\title{
There is level 1 evidence for intensive glycemic control for reducing the progression of diabetic retinopathy in persons with type 2 diabetes
}

\author{
Emily Y. Chew
}

Received: 2 February 2015/Accepted: 12 February 2015/Published online: 27 February 2015

(C) Springer Science+Business Media New York (outside the USA) 2015

Researchers of several medical departments of an academic institution conducted the meta-analysis of studies that evaluated the role of glycemic control in diabetic ocular complications that appears in the current issue of this journal (e-published in October 2014) [1]. Their final conclusion was that more clinical trials are needed to conclusively evaluate the role of glycemic control in ocular complications particularly in persons with type 2 diabetes. I respectfully disagree and counter their argument with discussion regarding study design and the use of surrogate outcomes in studies of diabetic retinopathy; and offer other interpretation of the previous studies. Clinical trial results provide important guidance for care and may not answer all clinical questions posed but there is sufficient level 1 evidence to recommend intensive glycemic control now for persons with type 2 diabetes for the most common and important ocular complications, progression of diabetic retinopathy.

I agree with the authors that without a doubt, functional outcomes are most important for the patients. Patients are the ones who suffer from vision loss, reducing their ability to read, drive, and perform other activities of daily living. It is important to point out that prior to the common use of lasers, $50 \%$ of all patients who developed proliferative diabetic retinopathy, the most severe form of diabetic retinopathy, would be legally blind in 5 years $[2,3]$. Ophthalmologists practicing before the 1970s described their eye clinics being filled with persons suffering from

E. Y. Chew ( $\square)$

Division of Epidemiology and Clinical Applications, National Eye Institute/National Institutes of Health, Building 10, CRC, Room 3-2531, 10 Center Drive, MSC-1204, Bethesda, MD 20892-1204, USA

e-mail: echew@nei.nih.gov diabetes who were often accompanied by their seeing-eye dogs. With the onset of laser photocoagulation and the results of the original Diabetic Retinopathy Study [4], this rate of severe vision was reduced initially by $50 \%$ but with further refinement of the laser treatment strategies in the Early Treatment Diabetic Retinopathy Study (ETDRS) [5], the rate of severe vision loss was reduced by $95 \%$ [6], changing this scenario dramatically. This is a highly successful therapy for eradicating severe vision loss in persons with diabetic retinopathy.

The studies, which were conducted following the success of the laser photocoagulation, considered visual acuity changes to be secondary outcomes. Even in the highly successful Diabetes Control and Complications Trial (DCCT) [7], visual acuity change was NOT a primary outcome. It was the progression of diabetic retinopathy, along the ETDRS scale [8] which is a classification of diabetic retinopathy severity, a well characterized and highly validated scale for the measurement of diabetic retinopathy. The Food and Drug Administration (FDA) in the United States has accepted the progression along the ETDRS scale as a surrogate outcome for all clinical trials of diabetic retinopathy. A surrogate outcome is an outcome that specific treatment may affect. This surrogate outcome may correlate with a real clinical endpoint, including vision loss in this case. Progression along the scale is a sign of deterioration and eventually, patients develop vision loss from the diabetic retinopathy and may need laser photocoagulation or vitrectomy. A combined outcome of progression along the ETDRS scale and the treatment with laser photocoagulation or vitrectomy is also another outcome used in the clinical trials.

If we were to calculate the sample sizes required to reduce the rate of vision loss, it may take tens of thousands of participants in each arm to find a beneficial (or adverse) 
effect because of the low event rate now of severe vision loss. Depending on the baseline severity of diabetic retinopathy, it might also take much longer to reach the meaningful clinical outcome of vision loss. For these reasons, the FDA has approved a number of studies using this surrogate outcome. The power calculations of these studies of glycemic control are based upon the rates of progression which eventually could lead to severe vision loss if we did not have such successful therapy.

As an investigator in the DCCT, a member of the Data and Safety Monitoring Committee of the Veterans Affair Diabetes Trial (VADT), and the Chair of the Actions to Control Cardiovascular Risk in Diabetes (ACCORD) Eye Study, I am familiar with the design issues of a number of studies of glycemic control for diabetic retinopathy. The ACCORD Trial, for example, had an overall sample size of over 10,000 participants. In the interest of using our funding judiciously, we calculated the sample size and found that the entire cohort was not required for the surrogate outcome of progression of diabetic retinopathy [9]. If we were to use visual acuity changes as an outcome, the 10,000 participants would not be enough to evaluate such a question. It is also important to note that we based our sample size upon data from the Wisconsin Epidemiologic Study of Diabetic Retinopathy (WESDR) [10], which was conducted several decades prior to the ACCORD Eye Study and estimated event rate was calculated to be $38.9 \%$ at 4 years, while the actual event rate of the ACCORD Eye Study was only about $10 \%$ [11]. This might be a reflection of the improved medical care, including better control of blood pressure as well as serum cholesterol, which have been proven to be important in the progression of diabetic retinopathy. This is an example of how event rates change as medical care and other characteristics change. Determining sample size may still be considered an art as event rates and surely the treatment effects are rarely known.

Fortunately, the ACCORD Eye Study confirmed the results of the UKPDS (United Kingdom Prospective Diabetes Study) that intensive glycemic control (and the use of fenofibrate with a statin) reduced the rate of progression of diabetic retinopathy in persons with type 2 diabetes [11]. The data from the ACCORD Eye Study were not used in the current meta-analysis to contribute to the success of glycemic control. Instead, the authors only used the outcomes of rates of vitrectomy and laser photocoagulation, which was only a small proportion of the entire outcome measurement. We have since published the secondary outcomes which may have been helpful to this analysis [12]. Nevertheless, the data from the main paper were sufficient to be included in this current meta-analysis.

Why did the other studies of glycemic control in persons with type 2 diabetes fail to show the important beneficial effects of glycemic control? Quite simply, there was probably a lack of power because of the inadequate sample size that had fundus photographs. It should be noted, however, that these smaller trials also reported non-significant results in the direction of a beneficial effect with intensive glycemic control [13-15]. Thus, the totality of evidence points toward the reduction of progression of diabetic retinopathy with intensive glycemic control compared with the standard care, as noted by the authors of the meta-analysis. But the task of proving reduction in vision loss would require trials with impractical huge numbers of participants as well as long duration of trials. The ACCORD Eye Study was about $\$ 8 \mathrm{M}$ while the entire ACCORD study which provided the infrastructure for the ancillary studies cost hundreds of millions of dollars. It is unlikely that we would have either the funding or the desire to launch such a study again. It would need further evaluation of other systemic changes to consider such a trial. In the meanwhile, I hope the arguments I have put forth provide evidence for the clinician to continue to work with patients with type 2 diabetes to reduce their risk of microvascular complications by maximizing their medical therapies. Patients are motivated to consider tight glycemic control to avoid blindness. Although fenofibrate has not been widely used by the internists, it may play an important role in the treatment of diabetic retinopathy, particularly in those who have evidence of diabetic retinopathy. We are also fortunate that persons with type 2 diabetes who are experiencing visual acuity loss due to diabetic macular edema have achieved significant visual acuity gain with effective intraocular injections of anti-vascular endothelial growth factor agents which have become the first line of therapy [16]. The era of requiring seeing-eye dogs is truly behind us.

Conflict of interest No financial disclosures (Emily Chew was an investigator in the Diabetes Control and Complications Trial (DCCT), member of the Data and Safety Monitoring Committee of the Veterans Affair Diabetes Trial (VADT), Chair of the Actions to Control Cardiovascular Risk in Diabetes (ACCORD) Eye Study).

\section{References}

1. X. Zhang, J. Zhao, T. Zhao, H. Liu, Effects of intensive glycemic control in ocular complications in patients with type 2 diabetes: a meta-analysis of randomized clinical trials. Endocrine (2015). doi:10.1007/s12020-014-0459-8

2. W.P. Beetham, Visual prognosis of proliferating diabetic retinopathy. Br. J. Ophtahlmol. 47, 611-619 (1963)

3. F.I. Caird, A.F. Burditt, G.J. Draper, Diabetic retinopathy: a further study of prognosis for vision. Diabetes 17, 121-128 (1968)

4. The Diabetic Retinopathy Study Research Group, Preliminary report on effects of photocoagulation therapy. Am. J. Ophthal. 81(4), 383-396 (1976) 
5. Early Treatment Diabetic Retinopathy Study Research Group, Early photocoagulation for diabetic retinopathy. ETDRS Report Number 9. Ophthalmology 98, 766-785 (1991)

6. F.L. Ferris 3rd, How effective are treatment for diabetic retinopathy? JAMA 269(10), 1290-1291 (1993)

7. The Diabetes Control and Complications Trial Research Group, The effect of intensive diabetes treatment on the progression of diabetic retinopathy in insulin dependent diabetes mellitus: the diabetes control and complications trial. Arch. Ophthalmol. 113, 36-51 (1995)

8. Early Treatment Diabetic Retinopathy Study Research Group, Fundus photographic risk factors for progression of diabetic retinopathy. ETDRS report number 12. Ophthalmology 98, 823-833 (1991)

9. E.Y. Chew, W.T. Ambrosius, L.T. Howard et al., Rationale, design, and methods of the Actions to Control Cardiovascular Risk in Diabetes Eye Study (ACCORD-EYE). Am. J. Cardiol. 99(12A), 103i-111i (2007)

10. R. Klein, B.E.K. Klein, S.E. Moss, K.J. Cruickshanks, Relationship of hyperglycemia to the long-term incidence and progression of diabetic retinopathy. Arch. Intern. Med. 154, 2169-2178 (1994)
11. The ACCORD Study Group and the ACCORD Eye, Study Group. Effects of medical therapies on retinopathy progression in type 2 diabetes. N. Engl. J. Med. 363(3), 233-244 (2010)

12. E.Y. Chew, M.D. Davis, R.P. Danis et al., The Effects of Medical Management on the progression of diabetic retinopathy in persons with type 2 diabetes: the action to control Cardiovascular Risk in Diabetes Eye Study. Ophthalmology 121(12), 2443-2451 (2014)

13. The ADVANCE Collaborative Group, Intensive blood glucose control and vascular outcomes in patients with type 2 diabetes. N. Engl. J. Med. 358, 2560-2572 (2008)

14. J.W. Beulens, A. Patel, J.R. Vingerling et al., Effects of blood pressure lowering and intensive glucose control on the incidence and progression of retinopathy in patients with type 2 diabetes mellitus: a randomized controlled trial. Diabetologia 52, 2027-2036 (2009)

15. W. Duckworth, C. Abraira, T. Moritz et al., Glucose control and vascular complications in veterans with type 2 diabetes. N. Eng. J. Med. 360, 129-139 (2009)

16. D.M. Brown, Q.D. Nguyen, D.M. Marcus et al., Long-term outcomes of ranibizumab therapy for diabetic macular edema: the 36-month results from two phase III trials: RISE and RIDE. Ophthalmology 120, 2013-2022 (2013) 